\title{
Meyniel's conjecture on the cop number: A survey
}

\author{
William Baird AND Anthony Bonato*
}

\begin{abstract}
Meyniel's conjecture is one of the deepest open problems on the cop number of a graph. It states that for a connected graph $G$ of order $n, c(G)=O(\sqrt{n})$. While largely ignored for over 20 years, the conjecture is receiving increasing attention. We survey the origins of and recent developments towards the solution of the conjecture. We present some new results on Meyniel extremal families containing graphs of order $n$ satisfying $c(G) \geq d \sqrt{n}$, where $d$ is a constant.

KEYWORDS AND PHRASES: Cops and robbers, cop number, retract, random graph.
\end{abstract}

\section{Introduction}

Cops and Robbers is a game played on a reflexive graph; that is, vertices each have at least one loop. Multiple edges are allowed, but make no difference to the game play, so we always assume there is exactly one edge between adjacent vertices. There are two players consisting of a set of cops and a single robber. The game is played over a sequence of discrete time-steps or rounds, with the cops going first in round 0 and then playing alternate timesteps. The cops and robber occupy vertices; for simplicity, we often identify the player with the vertex they occupy. We refer to the set of cops as $C$ and the robber as $R$. When a player is ready to move in a round they must move to a neighbouring vertex. Because of the loops, players can pass, or remain on their own vertex. Observe that any subset of $C$ may move in a given round.

The cops win if after some finite number of rounds, one of them can occupy the same vertex as the robber (in a reflexive graph, this is equivalent to the cop landing on the robber). This is called a capture. The robber wins if he can evade capture indefinitely. A winning strategy for the cops is a set of rules that if followed, result in a win for the cops. A winning strategy for the robber is defined analogously. Cops and Robbers is often called a vertex-pursuit game on graphs, for reasons that should now be apparent to the reader.

${ }^{*}$ The author gratefully acknowledge support from NSERC, Mprime, and Ryerson University. 
If we place a cop at each vertex, then the cops are guaranteed to win. Therefore, the minimum number of cops required to win in a graph $G$ is a well-defined positive integer, named the cop number (or copnumber) of the graph $G$. We write $c(G)$ for the cop number of a graph $G$. If $c(G)=k$, then we say $G$ is $k$-cop-win. In the special case $k=1$, we say $G$ is cop-win (or copwin).

The game of Cops and Robbers was first considered by Quilliot [22] in his doctoral thesis, and was independently considered by Nowakowski and Winkler [21]. The authors of [21] were told about the game by G. Gabor. Both [21, 22] refer only to one cop. The introduction of the cop number came in 1984 with Aigner and Fromme [1]. Many papers have now been written on cop number since these three early works; see the book [5] for additional references and background on the cop number. Cops and Robbers has even found recent application in robotics, artificial intelligence, and socalled moving target search; see [17, 19].

\subsection{Meyniel's conjecture}

Meyniel's conjecture states that if $G$ is a graph of order $n$, then

$$
c(G)=O(\sqrt{n})
$$

In other words, there is a constant $d>0$ such that

$$
c(G) \leq d \sqrt{n}
$$

We will refer to (1) as the Meyniel bound. The conjecture was mentioned in Frankl's paper [15] as a personal communication to him by Henri Meyniel in 1985 (see page 301 of [15] and reference [8]; see Figure 1 for a rare photograph of Meyniel). Despite this somewhat cryptic reference, Meyniel's conjecture stands out as one of the deepest (if not the deepest) problems on the cop number. The conjecture was largely unnoticed until recently, with several new works supplying upper bounds to the cop number or solving partial cases; see $[4,10,16,18,26,28]$. One of the motivations of this survey is to summarize what is currently known on the problem, while supplying the requisite background for researchers to consider its aspects (and solution!) in the future.

For $n$ a positive integer, let $c(n)$ be the maximum value of $c(G)$, where $G$ is of order $n$. For example, $c(1)=c(2)=c(3)=1$, while $c(4)=c(5)=2$. 


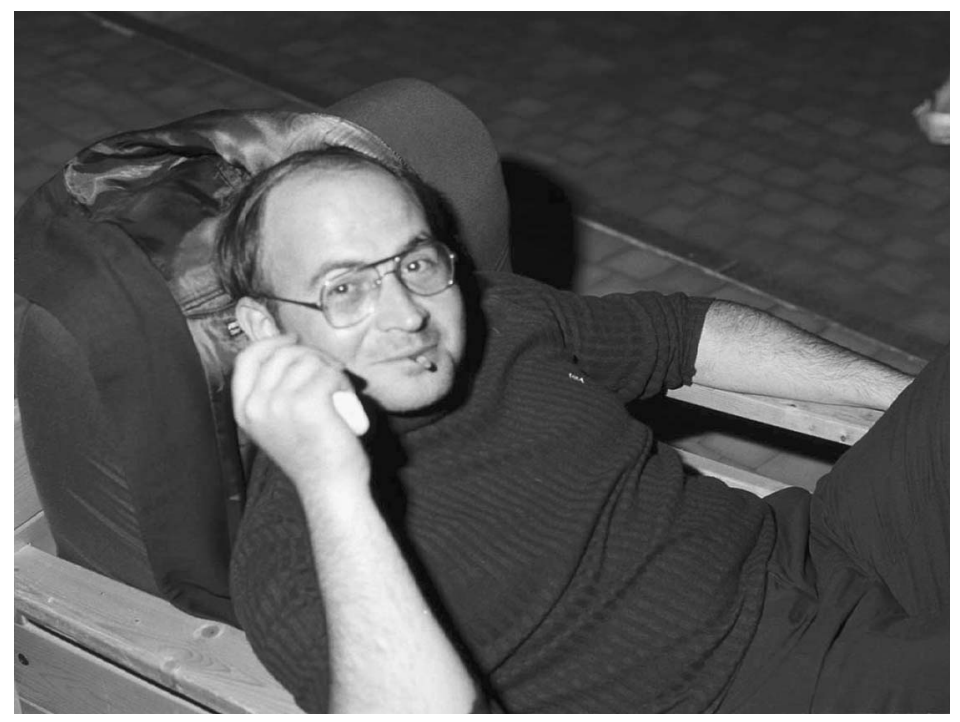

Figure 1: Henri Meyniel in Aussois, France, in the 1980's. Photo courtesy of Geňa Hahn.

Note that $c(n)$ is a non-decreasing function (to see this, note that adding a vertex of degree one does not change the cop number). We can rephrase Meyniel's conjecture more compactly as

$$
c(n)=O(\sqrt{n}) .
$$

At the heart of Meyniel's conjecture, of course, is the task of finding good upper bounds for the cop number. Incidence graphs of projective planes show that if the conjecture is true, then the bound is asymptotically tight (see Section 3). As a first step towards proving Meyniel's conjecture, Frankl [15] proved that $c(n)=o(n)$. Recent work has improved this upper bound somewhat (see Section 2). To further highlight how far we are from proving the conjecture, even the so-called soft Meyniel's conjecture is open, which states that for a fixed constant $c>0$,

$$
c(n)=O\left(n^{1-c}\right) .
$$

In Section 2 we give a history of upper bounds for the function $c(n)$. We close the section with a discussion of the conjecture in random graphs, in graph classes, and in directed graphs. We discuss families of graphs realizing the tightness of the Meyniel bound (1) in Section 3. 
For additional background and notation in graph theory, the reader is directed to the books $[11,30]$. All the graphs we consider are reflexive with no multiple edges, finite, and connected (for emphasis, we will occasionally remind the reader that the graphs under consideration are connected).

\section{Upper bounds for $c(n)$}

For many years, the best known upper bound was the one proved by Frankl [15].

Theorem 2.1. [15] For $n$ a positive integer

$$
c(n)=O\left(n \frac{\log \log n}{\log n}\right) .
$$

For a fixed integer $k \geq 1$, an induced subgraph $H$ of $G$ is $k$-guardable if, after finitely many moves, $k$ cops can move only in the vertices of $H$ in such a way that if the robber moves into $H$ at round $t$, then he will be captured at round $t+1$. For example, a clique or a closed neighbour set (that is, a vertex along with its neighbours) in a graph are 1-guardable.

Given a connected graph $G$, the distance between vertices $u$ and $v$ in $G$, denoted $d_{G}(u, v)$, is the length of a shortest path connecting $u$ and $v$. A path $P$ in $G$ is isometric if for all vertices $u$ and $v$ of $P$,

$$
d_{P}(u, v)=d_{G}(u, v)
$$

For example, a shortest path (or geodesic) connecting two vertices is isometric. The following theorem of Aigner and Fromme [1] on guarding isometric paths has found a number of applications.

Theorem 2.2. [1] An isometric path is 1-guardable.

For completeness, we give a proof of Frankl's upper bound (inspired by the discussion of Lu, Peng [18]) making use of the Moore bound, which is an important inequality involving the order $n$ of graph, its maximum degree $\Delta$, and its diameter. For simplicity, we will write $\operatorname{diam}(G)=D$.

Theorem 2.3. Let $G$ be a graph of order $n$, with maximum degree $\Delta>2$ and diameter $D$. Then

$$
n \leq 1+\Delta\left(\frac{(\Delta-1)^{D}-1}{\Delta-2}\right)
$$


Proof of Theorem 2.1. Each closed neighbour set of a vertex $u$ of maximum degree $\Delta$ is 1-guardable. By Theorem 2.2, an isometric path of length $D$ is also 1-guardable. Asymptotically, the Moore bound becomes $n=O\left(\Delta^{D}\right)$.

By the Moore bound, both $\Delta$ and $D$ cannot be less than $O\left(\frac{\log n}{\log \log n}\right)$. In particular, there is a subset $X$ consisting of either a closed neighbour set or isometric path of order at least $\frac{\log n}{\log \log n}$ in $G$. Delete $X_{1}$ to form the graph $G^{\prime \prime}$. Although graph $G^{\prime \prime}$ may be disconnected, the robber is confined to a connected component $G^{\prime}$ of this graph. The cops then move to $G^{\prime}$. Then

$$
c(G) \leq c\left(G^{\prime}\right)+1
$$

since $X_{1}$ is 1-guardable. Now proceed by induction using (3) to derive that

$$
\begin{aligned}
c(n) & \leq c\left(\frac{n}{2}\right)+\frac{n / 2}{\frac{\log n}{\log \log n}} \\
& =O\left(n \frac{\log \log n}{\log n}\right),
\end{aligned}
$$

where the equality follows by a straightforward induction.

The greedy approach used above in the proof of Frankl's theorem was used by Chinifooroshan [10] in 2008 to give an improved bound.

Theorem 2.4. [10] For $n$ a positive integer

$$
c(n)=O\left(\frac{n}{\log n}\right) .
$$

The bound (4), therefore, represents the first important step forward in proving Meyniel's conjecture in over 25 years. The key to proving (4) comes again from the notion of guarding an induced subgraph. A minimum distance caterpillar (or $m d c$ ) is an induced subgraph $H$ of $G$ with the following properties.

1. The graph $H$ is a tree.

2. There is a path $P$ in $H$ that is dominating: that is, for each vertex $u$ of $H$ not in $P$, there is a vertex $v$ of $P$ joined to $u$.

Figure 2 gives an example of a minimum distance caterpillar.

Mdc's are "sticky" analogues of isometric paths, and require just a few more cops to guard. The following theorem of [10] can be used to prove Theorem 2.4 in a way similar to the proof of Theorem 2.1 above. 


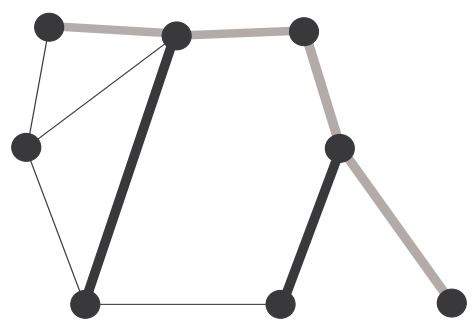

Figure 2: An example of an mdc, represented by the thicker lines. The grey lines forms the path $P$.

\section{Theorem 2.5. [10]}

1. An mdc is 5-guardable.

2. If $G$ has order $n$, then there is an $m d c$ in $G$ of order at least $\log n$.

Let $H$ be an induced subgraph of $G$. We say that $H$ is a retract of $G$ if there is a homomorphism $f$ from $G$ onto $H$ so that $f(x)=x$ for $x \in V(H)$; that is, $f$ is the identity on $H$. The map $f$ is called a retraction. Isometric paths are retracts in reflexive graphs: the cops stay on the image of the robber under the retraction. If the robber moves to the subgraph, then the cop captures the robber on his image or shadow there. One could imagine exploiting larger retracts in graphs as an approach to proving Meyniel's conjecture. Unfortunately, this will not substantially improve upper bounds on the cop number for general graphs. A recent result from [4] puts a polylogarithmic upper bound on the order of retracts in some graphs. The proof relies on the probabilistic method.

Theorem 2.6. [4] For all integers $n>0$, there is a graph of order $n$ whose largest retract is of order $O\left(\log ^{8} n\right)$.

An improvement exists to the bound (4) in Theorem 2.4. The following theorem was proved independently by three sets of authors.

Theorem 2.7. [18, 16, 28] For $n$ a positive integer

$$
c(n) \leq O\left(\frac{n}{2^{(1-o(1)) \sqrt{\log _{2} n}}}\right) .
$$

The bound in (5) is currently the best upper bound for general graphs that is known, but it is still far from proving Meyniel's conjecture or even the soft version of the conjecture. We note that the proofs of Theorem 2.7 
in $[18,28]$ use the greedy approach as in the proofs of Theorems 2.1 and 2.4, while expansion properties are used in [16]. In addition, all of the proofs use the probabilistic method, which represents a new and interesting approach to proving the conjecture.

\subsection{Random graphs}

As further support for its veracity, Meyniel's conjecture has been essentially proven for binomial random graphs $G(n, p)$. Let $p=p(n)$ be a function of $n$ with range in $[0,1]$. The probability space $\mathcal{G}(n, p)=(\Omega, \mathcal{F}, \mathbb{P})$ of random graphs is defined so that $\Omega$ is the set of all graphs with vertex set $[n], \mathcal{F}$ is the family of all subsets of $\Omega$, and for every $G \in \Omega$

$$
\mathbb{P}(G)=p^{|E(G)|}(1-p)^{\left(\begin{array}{c}
n \\
2
\end{array}\right)-|E(G)|} .
$$

The space $G(n, p)$ can be viewed as a result of $\left(\begin{array}{l}n \\ 2\end{array}\right)$ independent coin flips, one for each pair of vertices $\{x, y\}$, with the probability that $x$ and $y$ are joined equaling $p$. We will abuse notation and consider $G(n, p)$ as a graph, and so write $c(G(n, p))$ (note that the cop number is a random variable on the probability space $G(n, p)$ ). We say that an event holds asymptotically almost surely (a.a.s.) if it holds with probability tending to 1 as $n \rightarrow \infty$.

In 2009, Bollobás, Kun, Leader proved the following result [4], which proves Meyniel's bound in random graphs $G(n, p)$ up to a multiplicative logarithmic factor for a wide range of $p=p(n)$. The basic idea behind the proof is to surround the robber using Hall's theorem on matchings, and then use induction.

Theorem 2.8. If $p \geq 2.1 \log n / n$, then a.a.s.

$$
c(G(n, p))=O(\sqrt{n} \log n) .
$$

Recent work by Prałat and Wormald [26] removes the $\log n$ factor in (6) and hence, proves the Meyniel bound for random graphs (and also for random regular graphs).

\subsection{Graph classes}

While Meyniel's conjecture is unresolved for general graphs, we may attempt to solve it in certain graph classes. In some cases, the extra structure available in a class of graphs can bound the cop number from above more easily. For example, Aigner and Fromme [1] proved that $c(G) \leq 3$ if $G$ is planar. 
For a fixed graph $H$, Andreae [2] generalized this result by proving that the cop number of a $K_{5}$-minor-free graph (or $K_{3,3}$-minor-free graph) is at most 3 (recall that planar graphs are exactly those which are $K_{5}$-minor-free and $K_{3,3}$-minor-free). Andreae [3] also proved that for any graph $H$ the cop number of the class of $H$-minor-free graphs is bounded above by a constant.

Lu and Peng [18] show that the Meyniel bound holds in the class of graphs with diameter two. The proof uses the notion of guarding subgraph, but also uses a randomized argument.

Theorem 2.9. [18] If $G$ is a graph on $n$ vertices with diameter two, then

$$
c(G) \leq 2 \sqrt{n}-1 \text {. }
$$

The same bound (7) was also shown in [18] in the case when $G$ is bipartite and of diameter at most three.

The incidence graphs of projective planes are bipartite of diameter three, and so show that the bound (7) is asymptotically tight in that class. Meyniel extremal families whose members have diameter two and bounded chromatic number were given in [6].

\subsection{Directed graphs}

Another direction is the analogue of Meyniel's conjecture in digraphs. For the conjecture to be sensible, we should restrict our attention to strongly connected graphs (otherwise, a digraph can have cop number $n-1$ even if the underlying graph is connected). Recent work by Frieze et al. [16] using expansion properties shows that the cop number of a connected digraph of order $n$ is $O\left(n(\log \log n)^{2} / \log n\right)$. Can we do better? In other words, does the Meyniel bound hold for strongly connected digraphs? For tournaments, Meyniel's bound fails to be tight. A set $D$ is dominating in a tournament, if for each vertex $x$ not in $D$, there is a vertex $y$ in $D$ with $(y, x)$ a directed edge. The domination number of a tournament $G$, written $\gamma(G)$, is the minimum cardinality of a dominating set. Erdös proved (see p. 28 of [20]) that if $G$ is a tournament on $n$ vertices, then $\gamma(G) \leq\left\lceil\log _{2} n\right\rceil$, thereby giving a logarithmic upper bound on the cop number of tournaments.

\section{Lower bounds for $c(n)$}

Meyniel's conjecture states that the cop number is at most approximately $\sqrt{n}$. Examples are known (and will be discussed immediately below) which have cop number very close to $\sqrt{n}$. However, the question remains how close the cop number can approach $\sqrt{n}$ from below. 
For graphs with large cop number, we turn to incidence graphs. An incidence structure consists of a set $P$ of points, and a set $L$ of lines along with an incidence relation consisting of ordered pairs of points and lines. Given an incidence structure $S$, we define its incidence graph $G(S)$ to be the bipartite graph whose vertices consist of the points (one color), and lines (the second color), with a point joined to a line if it is incident with it in $S$. Incidence structures (and graphs) are quite general, but we restrict our attention to partial linear spaces, where any pair of points (lines) is incident with at most one line (point). It is an exercise that the incidence graph of a partial linear space has diameter at least three with girth at least 6 .

Projective planes are some of the most well-studied examples of incidence structures. A projective plane consists of a set of points and lines satisfying the following axioms.

1. There is exactly one line incident with every pair of distinct points.

2. There is exactly one point incident with every pair of distinct lines.

3. There are four points such that no line is incident with more than two of them.

Hence, projective planes are particular partial linear spaces; condition three rules out certain degenerate cases where all points are on a single line or all lines are on a single point. We are interested in finite projective planes, which always have $q^{2}+q+1$ points for some integer $q>0$ (called the order of the plane).

For a given projective plane $P$, define $G(P)$ to be the bipartite graph with red vertices the points of $P$, and the blue vertices represent the lines. Vertices of different colors are joined if they are incident. We call this the incidence graph of $P$. See Figure 3 for $G(P)$, where $P$ is the Fano plane (that is, the projective plane of order 2). We note the incidence graph of the Fano plane is isomorphic to the famous Heawood graph.

Aigner and Fromme proved the following theorem in [1], which provides a useful lower bound on the cop number in some graphs. The girth of a graph is the length of a shortest cycle. The minimum degree of $G$ is written $\delta(G)$.

Theorem 3.1. [1] If $G$ has girth at least 5 , then $c(G) \geq \delta(G)$.

Hence, Theorem 3.1 proves that $c(G(P)) \geq q+1$. As proven in [25], we actually have that $c(G(P))=q+1$. However, the orders of $G(P)$ depend on the orders of projective planes. The only orders where projective planes are known to exist are prime powers; indeed, this is a deep conjecture in finite geometry. What about integers which are not prime powers? An infinite family of graphs $\left(G_{n}: n \geq 1\right)$ is Meyniel extremal if there is a constant $d$ such that for all $n, c\left(G_{n}\right) \geq d \sqrt{\left|V\left(G_{n}\right)\right|}$. 

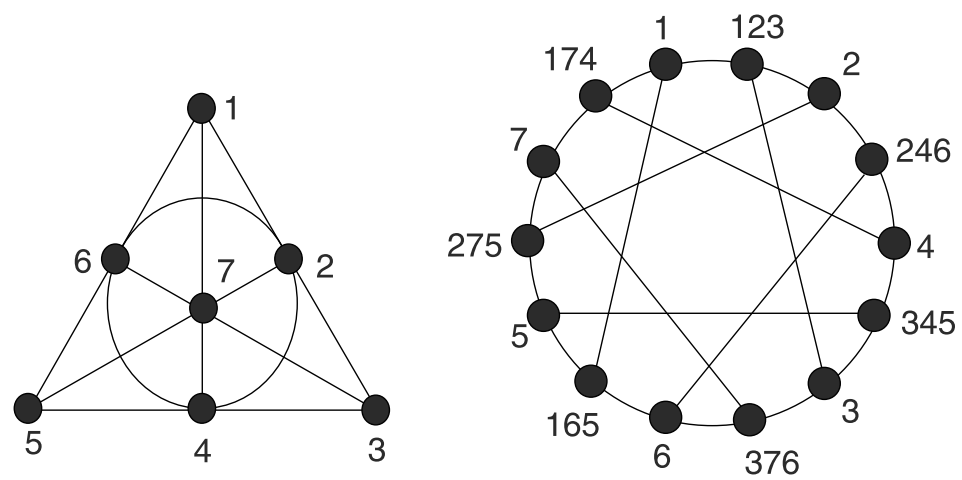

Figure 3: The Fano plane and its incidence graph. Lines are represented by triples.

Recall the famous Bertrand postulate (see [9, 12]).

Theorem 3.2. For all integers $x>1$, there is a prime in the interval $(x, 2 x)$.

In [25], a Meyniel extremal family was given using incidence graphs of projective planes and Theorem 3.2. Using Bertrand's postulate, it was shown that

$$
c(n) \geq \sqrt{\frac{n}{8}}
$$

for $n \geq 72$. Using this theorem and a result from number theory, it was shown in [25] that for sufficiently large $n$,

$$
c(n) \geq \sqrt{\frac{n}{2}}-n^{0.2625} .
$$

We do not know if (8) is the best possible lower bound for $c(n)$, and it would be interesting to find out.

A graph is $(a, b)$-regular if each vertex has degree either $a$ or $b$. We provide a new construction, giving infinitely many Meyniel extremal families containing graphs which are $(a, b)$-regular for certain $a$ and $b$. An affine plane of order $q$ has $q^{2}$-many points, each line has $q$ points, and each pair of distinct points is on a unique line. In an affine plane, there are $q^{2}+q$ lines, and each point is on $q+1$ lines. The relation of parallelism on the set of lines is an equivalence relation, and the equivalence classes are called parallel classes. Note that each parallel class contains $q$ lines, and there are $q+1$ parallel classes. 
Theorem 3.3. For a prime power $q$ and all $k=o(q)$, there exist graphs of order $2 q^{2}+(1-k) q$ which are $(q+1-k, q)$-regular and have cop number between $[q+1-k, q]$.

Note the graphs described in Theorem 3.3 have order $(1-o(1)) q^{2}$ with cop number $(1-o(1)) q$ and so are Meyniel extremal. In particular, we can set $k=q^{1-\varepsilon}$, for $\varepsilon \in(0,1)$ and obtain infinitely many distinct Meyniel families.

Proof of Theorem 3.3. Consider an affine plane $\mathcal{A}$ with order $q$. The incidence graph $G(\mathcal{A})$ has order $2 q^{2}+q$, and is $(q+1, q)$-regular. As $G(\mathcal{A})$ has girth at least 6 , we have that the graphs $\{G(\mathcal{A}): \mathcal{A}$ an affine plane of order $q$ \} form a Meyniel extremal family.

Affine planes of order $q$ may be partitioned into $(q+1)$-many parallel classes, each containing $q$ lines. Form the partial planes $\mathcal{A}^{-k}$ by deleting the lines in some fixed set of $k>0$ parallel classes. For a given $\mathcal{A}^{-k}$ the bipartite graph $G\left(\mathcal{A}^{-k}\right)$ is then $(q+1-k, q)$-regular, and has order $2 q^{2}+(1-k) q$. As the girth is at least 6 , we have by Theorem 3.1 that

$$
c(G) \geq q+1-k .
$$

We claim that

$$
c(G) \leq q .
$$

To prove (9), we play with $q$ cops. Fix a parallel class which was not deleted, say $\ell$, and place one cop on each line of the parallel class. As each point is on some line in $\ell$, the robber must move to some line $L \notin \ell$ to avoid being captured in the first round.

Fix a point $P$ of $L$, and let $L^{\prime}$ be the line of $\ell$ which intersects $L$ at $P$. Move the cop on $L^{\prime}$ to $P$. Now the robber cannot remain on $L$ without being captured, and so must move to some point. However, each point not on $L^{\prime}$ is joined to some cop, so the robber must move to a point of $L^{\prime}$. But the unique point on $L^{\prime}$ joined to $L$ is $P$, which is occupied by a cop.

Recent work in [6] provides constructions of new Meyniel extremal families from designs and geometries.

\section{Acknowledgements}

The author thanks Graeme Kemkes, Richard Nowakowski, and Pawel Prałat for helpful discussions. 


\section{References}

[1] Aigner, M. and Fromme, M. (1984). A game of cops and robbers. Discrete Applied Mathematics 8 1-12. MR0739593

[2] Andreae, T. (1984). Note on a pursuit game played on graphs. Discrete Applied Mathematics 9 111-115. MR0761595

[3] Andreae, T. (1986). On a pursuit game played on graphs for which a minor is excluded. J. Combin. Th., Ser. B 41 37-47. MR0854602

[4] Bollobás, B., Kun, G. and Leader, I. (2012). Cops and robbers in a random graph. Preprint.

[5] Bonato, A. and Nowakowski, R. J. (2011). The Game of Cops and Robbers on Graphs. American Mathematical Society, Providence, Rhode Island. MR2830217

[6] Bonato, A. and Burgess, A. (2012). Cops and robbers on graphs based on designs. Preprint.

[7] Bonato, A., Chiniforooshan, E. and Prałat, P. (2010). Cops and robbers from a distance. Theoretical Computer Science 411 3834-3844. MR2779754

[8] Bonato, A., Kemkes, G. and Prałat, P. (2012). Almost all cop-win graphs contain a universal vertex. Discrete Mathematics 312 16521657.

[9] Chebyshev, P. (1850). Mémoire sur les nombres premiers. Mém. Acad. Sci. St. Pétersbourg 7 17-33.

[10] Chiniforooshan, E. (2008). A better bound for the cop number of general graphs. Journal of Graph Theory 58 45-48. MR2404040

[11] Diestel, R. (2000). Graph Theory. Springer-Verlag, New York. MR1743598

[12] Erdős, P. (1930-32). Beweis eines Satzes von Tschebyschef. Acta Sci. Math. (Szeged) 5 194-198.

[13] Erdős, P. and Rényi, A. (1959). On random graphs I. Publicationes Mathematicae Debrecen 6 290-297. MR0120167

[14] Erdős, P. and Rényi, A. (1960). On the evolution of random graphs. Publ. Math. Inst. Hungar. Acad. Sci. 5 17-61. MR0125031

[15] Frankl, P. (1987). Cops and robbers in graphs with large girth and Cayley graphs. Discrete Applied Mathematics 17 301-305. MR0890640 
[16] Frieze, A., Krivelevich, M. and Loh, P. (2012). Variations on cops and robbers. Journal of Graph Theory 69 383-402.

[17] Isaza, A., Lu, J., Bulitko, V. and Greiner, R. (2008). A cover-based approach to multi-agent moving target pursuit. In: Proceedings of The 4th Conference on Artificial Intelligence and Interactive Digital Entertainment.

[18] Lu, L. and Peng, X. (2012). On Meyniel's conjecture of the cop number. Preprint.

[19] Moldenhauer, C. and Sturtevant, N. (2009). Evaluating strategies for running from the cops. In: Proceedings of IJCAI.

[20] Moon, J. W. (1968). Topics on Tournaments. Holt, Rinehart and Winston, New York. MR0256919

[21] Nowakowski, R. J. and Winkler, P. (1983). Vertex-to-vertex pursuit in a graph. Discrete Mathematics 43 235-239. MR0685631

[22] Quilliot, A. (1978). Jeux et pointes fixes sur les graphes. Thèse de 3ème cycle. Université de Paris VI, pp. 131-145.

[23] Quilliot, A. (1983). Problèmes de jeux, de point Fixe, de connectivité et de represésentation sur des graphes, des ensembles ordonnés et des hypergraphes. Thèse d'Etat, Université de Paris VI, pp. 131-145.

[24] Quilliot, A. (1985). A short note about pursuit games played on a graph with a given genus. J. Combin. Theory (B) 38 89-92. MR0782627

[25] Prałat, P. (2010). When does a random graph have constant cop number? Australasian Journal of Combinatorics 46 285-296. MR2598712

[26] Prałat, P. and Wormald, N. (2012). Meyniel's conjecture holds in random graphs. Preprint.

[27] Schroeder, B. S. W. (2001). The copnumber of a graph is bounded by $\left\lfloor\frac{3}{2}\right.$ genus $\left.(G)\right\rfloor+3$, Categorical perspectives (Kent, OH, 1998). Trends Math. Birkhäuser Boston, Boston, MA, pp. 243-263. MR1827672

[28] Scott, A. and Sudakov, B. (2011). A new bound for the cops and robbers problem. SIAM J. of Discrete Math. 25 1438-1442. MR2837608

[29] Sloane, N. J. A. (2012). Sequences A000088 and A001349. The On-Line Encyclopedia of Integer Sequences. Published electronically at http://oeis.org.

[30] West, D. B. (2001). Introduction to Graph Theory, 2nd edition. Prentice Hall. MR1367739 
William BAIRD

Department of Mathematics

RYERSON UNIVERSITY

Toronto, ON

Canada, M5B 2K3

E-mail address: w3baird@ryerson.ca

Anthony Bonato

Department of Mathematics

RYERSON UNIVERSITY

Toronto, ON

Canada, M5B 2K3

E-mail address: abonato@ryerson.ca

ReCEIVED JANuARY 12, 2012 